\title{
Desmopressin Acetate in Intracranial Haemorrhage
}

\author{
Thomas Kapapa, ${ }^{1}$ Stefan Röhrer, ${ }^{1}$ Sabine Struve, ${ }^{2}$ Matthias Petscher, ${ }^{3}$ Ralph König, \\ Christian Rainer Wirtz, ${ }^{1}$ and Dieter Woischneck ${ }^{4}$
}

\author{
${ }^{1}$ Neurochirurgische Klinik, Universitätsklinikum Ulm, Albert-Einstein-Allee 23, 89081 Ulm, Germany \\ ${ }^{2}$ Klinik für Hämatologie, Onkologie, Immunologie, Palliativmedizin, Infektiologie und Tropenmedizin, \\ Städtisches Klinikum München, Klinikum Schwabing, Kölner Platz 1, 80804 München, Germany \\ ${ }^{3}$ Klinik für Anästhesiologie, Abteilung Klinische Anästhesiologie, Albert-Einstein-Allee 23, 89081 Ulm, Germany \\ ${ }^{4}$ Neurochirurgische Klinik, Klinikum Landshut, Robert-Koch-Strasse 1, 84034 Landshut, Germany \\ Correspondence should be addressed to Thomas Kapapa; thomas.kapapa@uniklinik-ulm.de
}

Received 29 August 2014; Revised 28 November 2014; Accepted 8 December 2014; Published 23 December 2014

Academic Editor: Jeff Bronstein

Copyright (C) 2014 Thomas Kapapa et al. This is an open access article distributed under the Creative Commons Attribution License, which permits unrestricted use, distribution, and reproduction in any medium, provided the original work is properly cited.

\begin{abstract}
Introduction. The secondary increase in the size of intracranial haematomas as a result of spontaneous haemorrhage or trauma is of particular relevance in the event of prior intake of platelet aggregation inhibitors. We describe the effect of desmopressin acetate as a means of temporarily stabilising the platelet function. Patients and Methods. The platelet function was analysed in 10 patients who had received single $(N=4)$ or multiple $(N=6)$ doses of acetylsalicylic acid and 3 patients (control group) who had not taken acetylsalicylic acid. All subjects had suffered intracranial haemorrhage. Analysis was performed before, half an hour and three hours after administration of desmopressin acetate. Statistical analysis was performed by applying a level of significance of $P \leq 0.05$. Results. (1) Platelet function returned to normal 30 minutes after administration of desmopressin acetate. (2) The platelet function worsened again after three hours. (3) There were no complications related to electrolytes or fluid balance. Conclusion. Desmopressin acetate can stabilise the platelet function in neurosurgical patients who have received acetylsalicylic acid prior to surgery without causing transfusion-related side effects or a loss of time. The effect is, however, limited and influenced by the frequency of drug intake. Further controls are needed in neurosurgical patients.
\end{abstract}

\section{Introduction}

Platelet aggregation inhibitors such as acetylsalicylic acid, adenosine diphosphate (ADP) receptor inhibitors, and vita$\min \mathrm{K}$ antagonists are a fixed constant in the treatment of acute and chronic atherothrombotic vascular diseases, including myocardial infarction, acute coronary syndrome, peripheral arterial obstructive disease, and atrial fibrillation $[1,2]$. The prescribing data for platelet aggregation inhibitors and sales figures for acetylsalicylic acid suggest that their use is becoming more widespread (Figure 1) [3]. Their prescription and use are dependent on comorbidities and age, however, which can increase the attendant risk of haemorrhage [1].

These drugs, which are being used increasingly in the elderly, are of neurological importance in the event of intracranial haemorrhage, for instance. The types of intracranial haemorrhage deserving special mention are intracerebral, acute, and chronic subdural haemorrhage, and spontaneous subarachnoid haemorrhage.

Approximately $20 \%$ of all patients admitted with traumatic brain injury are found to have acute subdural haematoma, which primarily occurs between the age of 31 and 47 years. The mortality rate can exceed 75\% [4]. Out of 100,000 patients, 10-30 suffer spontaneous intracerebral haemorrhage. The incidence of intracerebral haemorrhage associated with strokes is approximately $15 \%$. The elderly are more likely to suffer intracerebral haemorrhage than younger individuals. The treatment outcome depends on the extent and localisation of the haemorrhage [5]. Chronic subdural haematoma (Figure 2) is one of the most common forms of traumatic intracranial haemorrhage [6] and is often caused by a mild traumatic incident only seldom recalled by the patient [7]. Common to all these types of haemorrhage is the fact that 


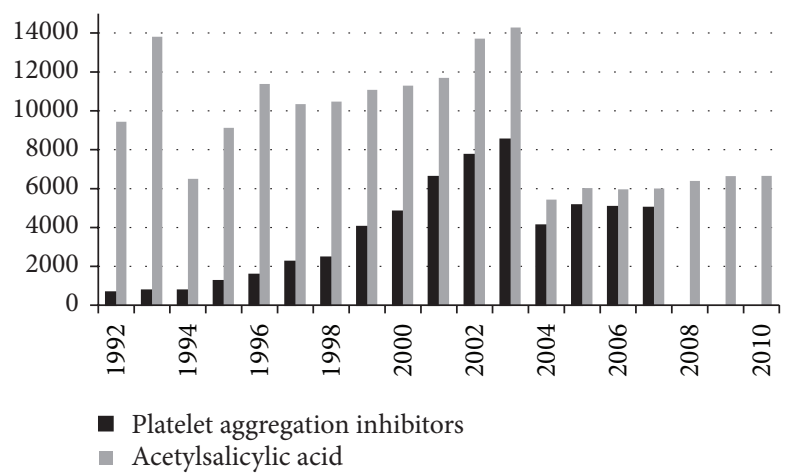

FIGURE 1: Drug prescribing: platelet aggregation inhibitor according to the German Rote Liste and generic drug: acetylsalicylic acid prescribed at the expense of the statutory health insurance funds [3].

more extensive and prolonged bleeding, as well as a greater risk of secondary haemorrhaging, can be expected if the patient is already taking a platelet aggregation inhibitor $[8,9]$ (Figure 3).

When faced with the dilemma of prohemostatic measures for minimising the complications of intracranial haemorrhage and an indication for platelet aggregation inhibition, the question of the best possible treatment arises. Desmopressin acetate, which is known to attenuate the effect of acetylsalicylic acid, has been studied in cardiac diseases [10-14]. Desmopressin (1-deamino-8-d-arginine vasopressin) increases plasma factor levels due to the endogenous release of coagulation factor VIII (FVIII), von Willebrand factor (vWF) and tissue plasminogen activator (tPA), enhanced platelet adhesion, and reduced bleeding time $[10-13,15]$. The exact mechanism of action of acetylsalicylic acid, however, has not been fully explained.

Here we discuss the possibility of attenuating the effect of acetylsalicylic acid based on an observational study in patients with intracranial haemorrhage.

\section{Patients and Methods}

The local ethics committee approved the laboratory and haematological tests in patients with traumatic brain injury and impaired consciousness.

2.1. Patients. Between 2008 and 2014, patients who had been admitted for acute intracranial haemorrhage and were already using acetylsalicylic acid, along with control patients, were enrolled in the study. Enrolment depended on the possibility to perform laboratory tests and so took place only during the day rather than at night and also depended on the availability of suitable patients, who were selected if they were available for analysis between 8.00 a.m. and 3.30 p.m. In addition, patients were only enrolled by three neurosurgical members of the study team.

The inclusion criteria required patients to be over 18 years of age and have a diagnosis of isolated spontaneous or traumatic intracranial haemorrhage confirmed by computed tomography. The haemorrhage alone had to be a correlate of

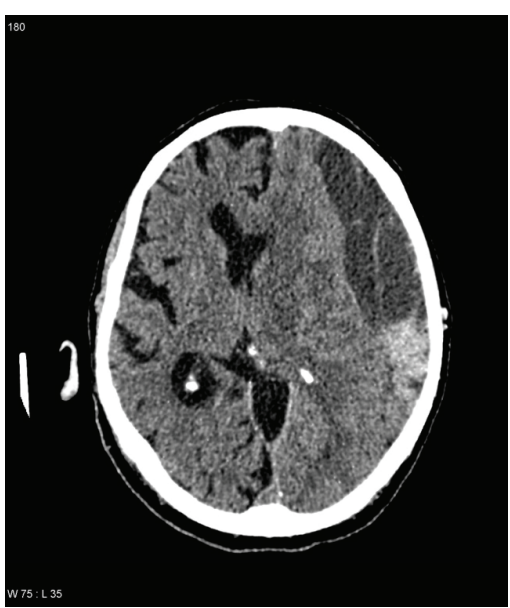

FIGURE 2: Computed tomography of a left-sided chronic subdural haematoma.

a neurological deficit such as coma, hemiparesis, or aphasia and thus presents a surgical indication, or the progressive size had to suggest that a clinically relevant deterioration was likely (compression of basal cisterns, midline shift). A further inclusion criterion was evidence (communicated personally or in writing) of the intake of acetylsalicylic acid within 24 hours prior to admission.

The exclusion criteria comprised intake of other anticoagulants or platelet aggregation inhibitors, known coagulation disorders, alcoholism, known hypercoagulable tendency, renal failure, hypothermia $\left(<35^{\circ} \mathrm{C}\right)$, multiple trauma, or polytrauma.

In doing so, a distinction was made between patients having taken acetylsalicylic acid once within 24 hours prior to admission ( Pat $_{\text {SIN }}$ ) and those having taken the drug regularly (daily) or more than once (Pat $\left.{ }_{M U L}\right)$. Patients who were conscious could be questioned; otherwise, information was taken from the records of other hospitals, documentation from previous stays at our hospital, or documentation obtained from a care facility or the general practitioner. Patient details are provided in Table 1.

The control group comprises 3 patients (one woman aged 52 years with spontaneous subarachnoid haemorrhage; one man aged 79 years with traumatic brain injury; one man aged 56 years with traumatic brain injury). Since-unlike the patients with intracranial haematoma-predominantly normal values were found initially and the values for platelet aggregation inhibition were normal, no further blood tests were performed over the course.

All patients were monitored intensively for intracranial haemorrhage and their potential (cardiac and circulatory) risks following administration of desmopressin.

2.2. Desmopressin Acetate and Platelet Function Tests. There are numerous recommendations to also use desmopressin acetate as a treatment for platelet function disorders or mild thrombocytopenia. Desmopressin acetate is used to improve 


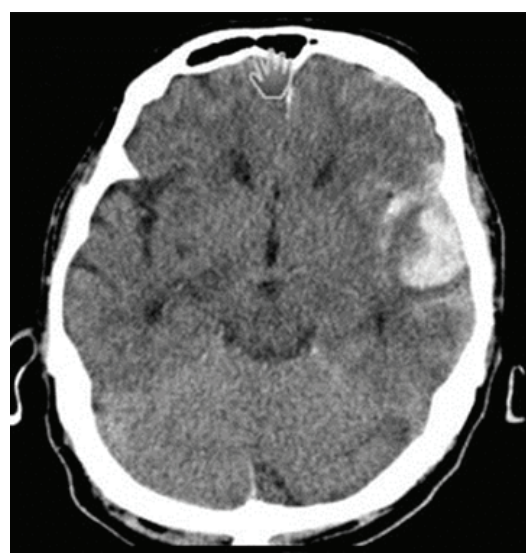

(a)

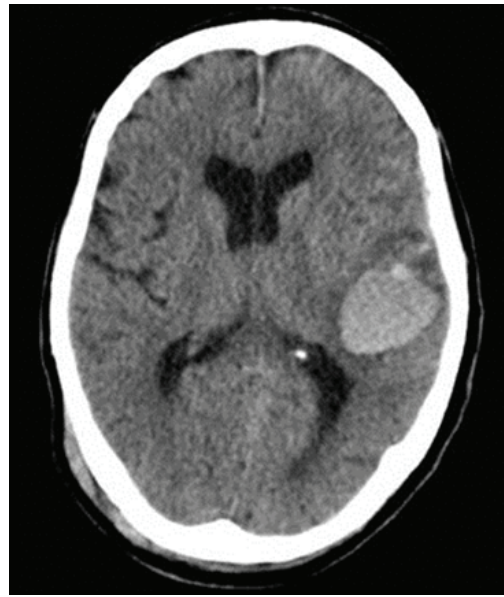

(c)

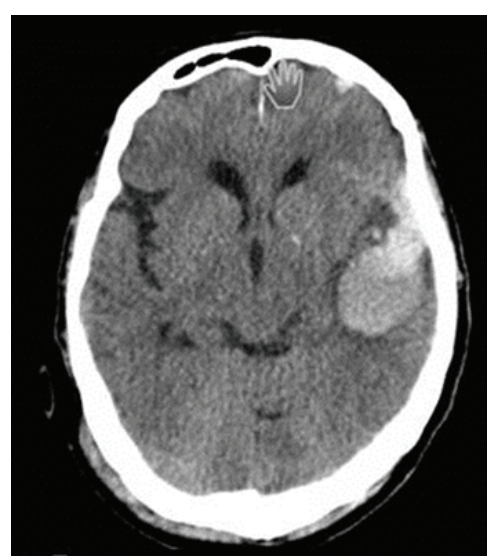

(b)

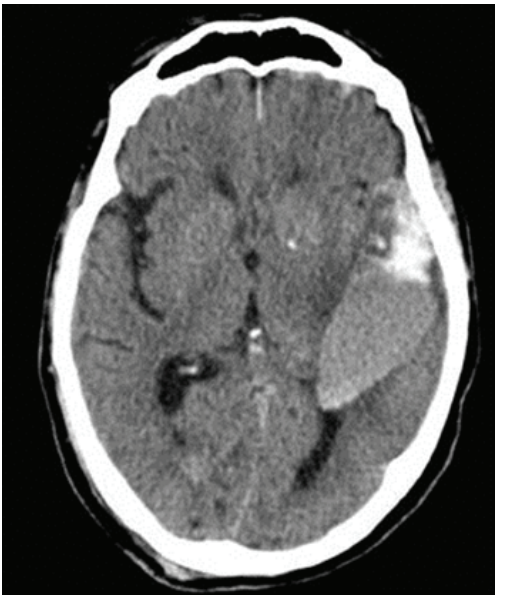

(d)

FIGURE 3: Spontaneous course of traumatic intracerebral haemorrhage with platelet aggregation inhibitor: 79-year-old patient hit by a car when walking in the city. On admission, he had a Glasgow Coma Score of 13. Findings on admission were left temporal contusion (a) which had increased in size after 24 hours (b). The patient then developed dysphasia. Although there was only a slight increase in size after a further 4 days, with the clinical condition remaining stable (c), an additional, relevant progression in size was noted on day 5 in the presence of seizures (d). Surgery was not performed since the patient never lost consciousness and did not develop severe hemiparesis.

primary haemostasis - not because of the enhanced aggregability of the platelets, but rather due to their increased adhesiveness [16].

Platelet function was tested using platelet function analysis (PFA-100, Dade Behring, Germany), which measures the time required for a platelet plug to form and seal a semipermeable membrane. This membrane is coated in collagen. As under physiological conditions, it serves as an initial matrix for platelet adhesion in relation to the von Willebrand factor found in the tested blood. During the test, the platelets bind directly with the collagen, which in turn leads to platelet activation. Adherent platelets are degranulated on contact with the agonists' epinephrine and adenosine phosphate, which are also found in the membrane. Activation of free-flowing platelets and blood clot aggregation is the outcome. Consequently, the opening in the membrane gradually closes (Figure 4) [17]. The standard values for membrane closure range from 68 to 120 seconds for adenosine phosphate and

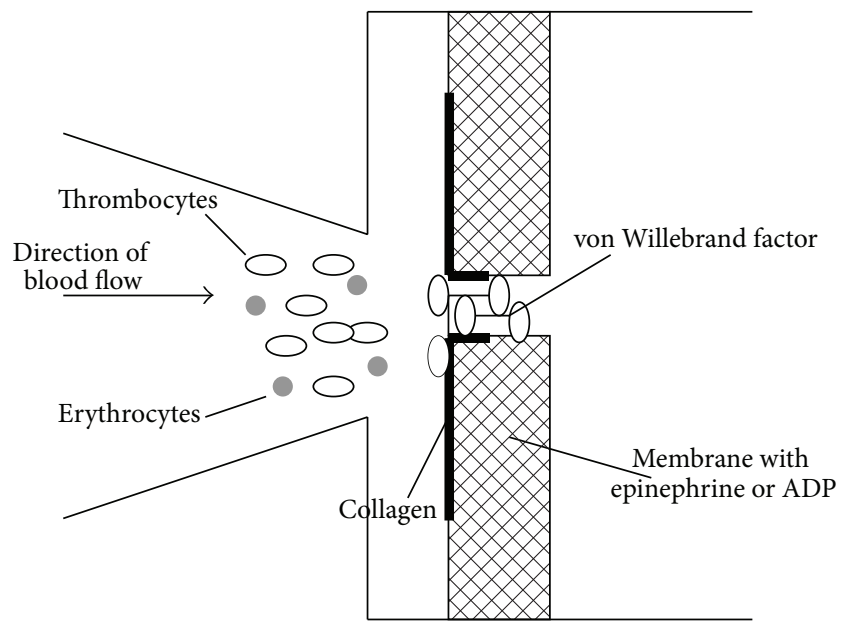

FIGURE 4: Functional principle of platelet function analysis 100 (PFA100) [17]. 
TABLE 1: Characteristics of the patient population. Cerebral infarction $=\mathrm{CI}$, spontaneous subarachnoid haemorrhage $=\mathrm{SAH}$, spontaneous intracerebral haemorrhage $=\mathrm{ICH}$, chronic subdural haematoma $=\mathrm{cSDH}$, traumatic brain injury $=\mathrm{TBI}$, and sodium $=\mathrm{mmol} / \mathrm{L}$.

(a) Multiple doses

\begin{tabular}{lcccccc}
\hline Gender & Age & Weight $(\mathrm{kg})$ & Height $(\mathrm{cm})$ & Cause & Sodium (prior/after) & Surgery \\
\hline M & 72 & 85 & 179 & ICH & $139 / 138$ & Yes \\
M & 52 & 73 & 179 & TBI & No \\
M & 72 & 85 & 180 & ICH & $134 / 140$ & Yes \\
M & 72 & 81 & 164 & ICH & $139 / 139$ & No \\
M & 52 & 65 & 179 & cSDH & $138 / 133$ \\
M & 66 & 89 & 160 & CI & Yes \\
\hline
\end{tabular}

(b) Single dose

\begin{tabular}{lcccccc}
\hline Gender & Age & Weight $(\mathrm{kg})$ & Height $(\mathrm{cm})$ & Cause & Sodium (prior/after) & Surgery \\
\hline F & 44 & 59 & 165 & CI & $137 / 137$ & Yes \\
M & 49 & 80 & 180 & SAH & TBI & $132 / 132$ \\
M & 76 & 70 & 175 & TSI & No \\
M & 80 & 80 & 170 & cSDH & $132 / 142$ & Yes \\
\hline
\end{tabular}

(c) Control group

\begin{tabular}{lcccccc}
\hline Gender & Age & Weight $(\mathrm{kg})$ & Height $(\mathrm{cm})$ & Cause & Sodium & Surgery \\
\hline M & 79 & 87 & 174 & TBI & 142 & No \\
M & 56 & 79 & 176 & TBI & 138 & No \\
F & 52 & 62 & 168 & SAH & 136 & Yes \\
\hline
\end{tabular}

54 to 165 seconds for epinephrine. The uneventful use of PFA100 in neurosurgical patients is documented in the literature [18].

2.3. Test Points. Platelet function was analysed prior to administration of desmopressin (T1). Desmopressin acetate was administered over a period of 30 minutes so as to avoid flushing and hypotension. The platelet function was then analysed half an hour (T2) and three hours (T3) after administration. All patients were given $24 \mu \mathrm{g}$ in $100 \mathrm{~mL}$ physiological saline solution over a period of 30 minutes. The time between sampling and starting the test was less than 15 minutes and did not hinder the procedures with respect to potential surgery.

Statistical analysis comprised the Wilcoxon and MannWhitney $U$ tests, with a significance level of $P \leq 0.05$.

\section{Results}

During the specified period, 10 patients on acetylsalicylic acid with the following conditions were enrolled: intracerebral haemorrhage $(N=3)$, cerebral infarctions $(N=2)$, traumatic brain injury with contusion or acute subdural haematoma $(N=2)$, spontaneous subarachnoid haemorrhage with hydrocephalus $(N=1)$, and chronic subdural haemorrhage $(N=2)$. Acetylsalicylic acid had been taken once by 4 patients and multiple times by 6 patients. Three patients were recruited as controls (Table 1 ). Seven of these 10 patients underwent surgery immediately after admission (Pat SIN $_{\text {S }} N=$ 3, Pat $_{\text {MUL }} N=4$ ); the remaining 3 patients were monitored under intensive care conditions.
The exact dose of acetylsalicylic acid could not always be determined, especially in those patients having taken only one dose. In the others, the dose did not exceed $100 \mathrm{mg}$.

(1) The platelet function was found to be impaired in both patient groups $\left(\mathrm{Pat}_{\mathrm{SIN}}\right.$ and $\left.\mathrm{Pat}_{\mathrm{MUL}}\right)$ at T1. Figures 5 and 6 illustrate the mean membrane occlusion times for time point $\mathrm{T} 1$, with results exceeding the norm of 120 seconds (adenosine phosphate) and 165 seconds (epinephrine).

(i) In the adenosine phosphate samples, the mean time at T1 was 122.7 seconds (SD 21.8) for Pat ${ }_{\mathrm{SIN}}$ and 126.6 seconds (SD 89.0) for $\operatorname{Pat}_{\mathrm{MUL}}(P=$ $0.394)$.

(ii) The epinephrine samples revealed a mean occlusion time at $\mathrm{T} 1$ of 249.5 seconds (SD 59.94) for Pat $_{\text {SIN }}(P=0.068)$.

(2) The mean value at $\mathrm{T} 1$ in the epinephrine tests was more than 300 seconds for Pat ${ }_{\mathrm{MUL}}$ (Figure 5).

(3) Platelet function had returned to normal or close to normal in both groups at T2 after administration of desmopressin acetate (Figures 5 and 6).

(i) In the adenosine phosphate samples, the mean time at T2 was 86.3 seconds (SD 32.06) for Pat SIN $_{\text {S }}$ and 73.83 seconds (SD 9.45) for $\operatorname{Pat}_{\mathrm{MUL}}(P=$ $0.831)$.

(ii) The epinephrine samples revealed a mean occlusion time at $\mathrm{T} 2$ of 170.5 seconds (SD 92.16) for 


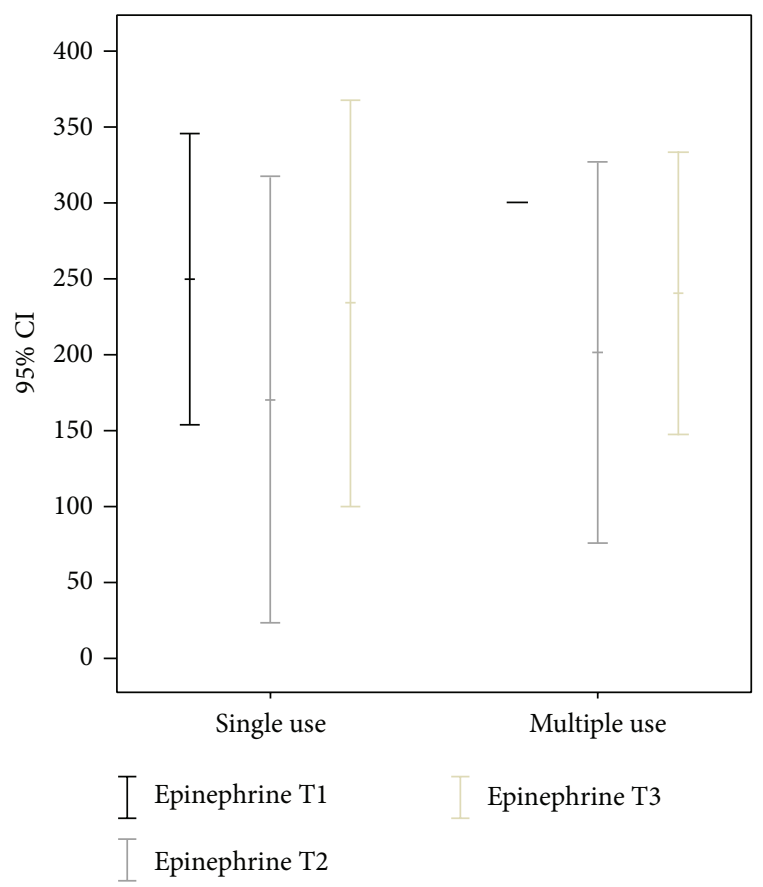

FIGURE 5: Results of platelet function analysis using epinephrine prior to administration (T1), half an hour after administration (T2), and three hours after administration (T3); $y$-axis in seconds.

Pat $_{\text {SIN }}$ and 192.8 seconds (SD 92.86) for Pat ${ }_{\text {MUL }}$ $(P=0.829)$.

(4) The platelet function was again impaired in both groups at T3, though the values were worse in the Pat ${ }_{\mathrm{MUL}}$ group under epinephrine (Figures 5 and 6).

(i) In the adenosine phosphate samples, the mean time at T3 was 99.5 seconds (SD 30.73) for Pat ${ }_{\text {SIN }}$ and 86.3 seconds (SD 7.91) for $\mathrm{Pat}_{\mathrm{MUL}}(P=$ $0.829)$.

(ii) The epinephrine samples revealed a mean occlusion time at $\mathrm{T} 3$ of 234 seconds (SD 83.53) for Pat $_{\text {SIN }}$ and 212.7 seconds (SD 94.78) for Pat ${ }_{\text {MUL }}$ $(P=0.998)$.

(5) There were no relevant deviations from the reference values in the control group: mean ADP was $106 \mathrm{sec}-$ onds (SD 18.5) and epinephrine 135,6 seconds (SD 16.5). There was one case of a relevant, secondary increase in intracranial haemorrhage with clinical deterioration. Surgery was not, however, indicated at any time.

No intraoperative bleeding complications or fluid or electrolyte imbalances were observed. No blood transfusions or reoperations were performed in this small cohort. The mean serum sodium and potassium levels, as well as urine volume, remained within the reference range or remained clinically irrelevant and so did not necessitate intervention.

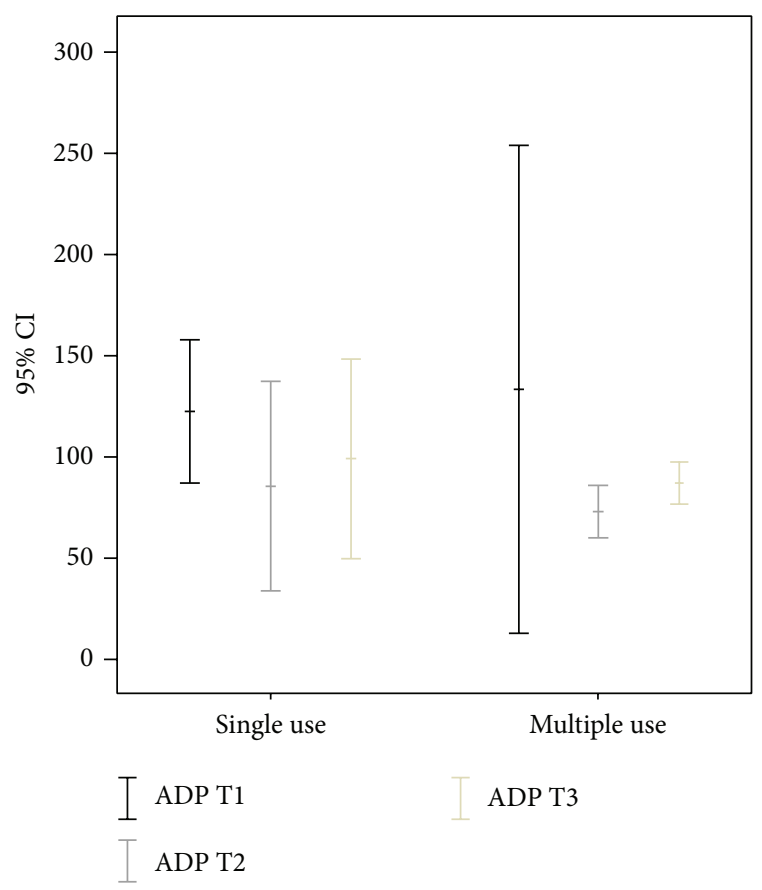

FIGURE 6: Results of platelet function analysis using adenosine phosphate (ADP) prior to administration (T1), half an hour after administration (T2), and three hours after administration (T3); $y$ axis in seconds.

\section{Discussion}

Taking traumatic brain injury as an example, there have been numerous reports of expansion in intracerebral haemorrhage within hours to days without the additional influence of external factors $[19,20]$. Impaired coagulation in traumatic brain injuries is reported at an incidence of up to $26 \%$ [21]. Survival and outcome after traumatic brain injury depend on any expansion in the preexisting haemorrhage [20]. Haemorrhagic volume also plays an influential role in survival in the case of spontaneous intracerebral haemorrhage. The more extensive the haemorrhage, the poorer the treatment outcome when measured on the Glasgow Outcome Scale [20].

Bleeding tendency can be increased by prior treatment with acetylsalicylic acid. Desmopressin acetate is capable of significantly reducing blood loss and thus minimising the haemorrhagic risk $[12,14,20]$.

As an antihemorrhagic agent with enhanced factor VIII coagulation activity, desmopressin acetate is authorised for use before surgery and tooth extraction and after traumas in mild to moderate haemophilia A and von-WillebrandJürgens disease [16]. However, paradigms are constantly shifting and there is still a shortage of data. There have been no reports of approval with respect to stabilising the platelet function in intracranial haemorrhage or prior to intracranial interventions following intake of acetylsalicylic acid. Aspirin has been identified as an independent predictor for death in patients suffering spontaneous intracranial haemorrhage [9].

We found that desmopressin acetate is a safe way to stabilise the platelet function in patients with intracranial haemorrhage if they are only taking acetylsalicylic acid. 
We were not able to determine the exact dose of acetylsalicylic acid or the exact time between intake and administration of desmopressin acetate. However, this matter displays daily clinical life in which decisions must be done on the basis of just little information. Hence, our results must be understood within mentioned conditions.

The platelet function tended to return to normal relatively rapidly, that is, after 30 minutes, using a dose of $24 \mu \mathrm{g}$. As a rule, this corresponds to the time it takes to admit the patient and prepare for emergency surgery. No electrolyte or fluid imbalances were found in our patient population. However, the platelet function deteriorates over the further course. Nevertheless, the question whether to administer desmopressin acetate immediately after diagnosing intracranial haemorrhage under acetylsalicylic acid or whether to only apply the drug preoperatively remains unanswered. Further studies must be conducted to verify the benefit of intranasal or intravenous administration of desmopressin acetate [22, 23].

We recommend administration of desmopressin in intracerebral haemorrhage so as to improve the coagulation status, as described in the literature $(20 \mathrm{~kg}$ body weight $=$ approximately $8 \mu \mathrm{g}, 50 \mathrm{~kg}$ body weight = approximately $20 \mu \mathrm{g}, 100 \mathrm{~kg}$ body weight $=40 \mu \mathrm{g}$, or $0.3 \mu \mathrm{g} / \mathrm{kg}$ body weight), followed by monitoring of the electrolyte and fluid balance $[16,24]$ in order to optimise the surgical setting.

Administration of platelet concentrates can be considered after applying desmopressin acetate; we would not recommend it, however, due to a lack of data [25-28]. Prolongation of normal platelet function is not immediately apparent as a result, and a more profound benefit would also appear doubtful given the fact that desmopressin acetate itself can stabilise the platelet function. Simultaneous administration of tranexamic acid should stabilise the blood clot, but further studies should be conducted [29].

\section{Conclusion}

Desmopressin acetate can improve the platelet function after 30 minutes in intracranial haemorrhage following acetylsalicylic acid intake. The coagulative status can thus be restored to normal levels in terms of platelet function for between 30 minutes and 3 hours. When administering desmopressin acetate in intracranial haemorrhage under acetylsalicylic acid, cardiac function, electrolyte, and fluid balance must be closely monitored.

\section{Disclosure}

Parts of the study were presented at the Annual Meeting of the German Society of Neurosurgeons 2011.

\section{Conflict of Interests}

All authors certify that they have no affiliations with or involvement in any organization or entity with any financial interest (such as honoraria; educational grants; participation in speakers' bureaus; membership, employment, consultancies, stock ownership, or other equity interest; and expert testimony or patent-licensing arrangements) or nonfinancial interest (such as personal or professional relationships, affiliations, knowledge, and beliefs) in the subject matter or materials discussed in this paper.

\section{Authors' Contribution}

Thomas Kapapa designed the study, included and treated patients, drafted the paper, and revised the paper. Stefan Röhrer included and treated patients and reviewed the paper. Sabine Struve designed and supervised the laboratory test concept. Matthias Petscher treated patients and revised the paper. Ralph König included patients in the study. Christian Rainer Wirtz interpreted the data and prepared and revised the paper. Dieter Woischneck designed the study, included patients, supervised the study, and reviewed the paper.

\section{References}

[1] D. J. Fintel, "Oral antiplatelet therapy for atherothrombotic disease: overview of current and emerging treatment options," Vascular Health and Risk Management, vol. 8, no. 1, pp. 77-89, 2012.

[2] J.-P. Bassand, "Review of atrial fibrillation outcome trials of oral anticoagulant and antiplatelet agents," Europace, vol. 14, no. 3, pp. 312-324, 2012.

[3] Federal Statistical Office of Germany, Prescibtion of Thrombocyte Aggregation Inhibitors in Germany, 2012, https://www.gbebund.de.

[4] M. R. Bullock, R. Chesnut, J. Ghajar et al., "Surgical management of acute subdural hematomas," Neurosurgery, vol. 58, no. 3, pp. S216-S224, 2006.

[5] M. Z. Raihan, M. H. Rashid, M. A. Syed, and M. H. Sarkar, "Factors influencing the surgical outcome of spontaneous intracerebral haematoma," Mymensingh Medical Journal, vol. 18, no. 2, pp. 245-249, 2009.

[6] M. Stanisic, M. Lund-Johansen, and R. Mahesparan, “Treatment of chronic subdural hematoma by burr-hole craniostomy in adults: influence of some factors on postoperative recurrence," Acta Neurochirurgica, vol. 147, no. 12, pp. 1249-1256, 2005.

[7] K.-M. Schebesch, C. Woertgen, R.-D. Rothoerl, O.-W. Ullrich, and A. T. Brawanski, "Cognitive decline as an important sign for an operable cause of dementia: chronic subdural haematoma," Zentralblatt für Neurochirurgie, vol. 69, no. 2, pp. 61-64, 2008.

[8] G. Baldi, F. Altomonte, M. Altomonte et al., "Intracranial haemorrhage in patients on antithrombotics: Clinical presentation and determinants of outcome in a prospective multicentric study in italian emergency departments," Cerebrovascular Diseases, vol. 22, no. 4, pp. 286-293, 2006.

[9] P. Saloheimo, M. Ahonen, S. Juvela, J. Pyhtinen, E.-R. Savolainen, and M. Hillbom, "Regular aspirin-use preceding the onset of primary intracerebral hemorrhage is an independent predictor for death," Stroke, vol. 37, no. 1, pp. 129-133, 2006.

[10] K.-H. Beck, P. Mohr, U. Bleckmann, H. Schweer, and V. Kretschmer, "Desmopressin effect on acetylsalicylic acid impaired platelet function," Seminars in Thrombosis and Hemostasis, vol. 21, supplement 2, pp. 32-39, 1995.

[11] K. H. Beck, U. Bleckmann, P. Mohr, V. Kretschmer, and B. Huss, "Shortening of acetylsalicylic acid-prolonged bleeding 
time by means of desmopressin," Beiträge zur Infusionstherapie und Transfusionsmedizin, vol. 32, pp. 437-439, 1994.

[12] D. P. Sheridan, R. T. Card, J. C. Pinilla et al., "Use of desmopressin acetate to reduce blood transfusion requirements during cardiac surgery in patients with acetylsalicylic-acid-induced platelet dysfunction," Canadian Journal of Surgery, vol. 37, no. 1, pp. 33-36, 1994.

[13] P. A. Flordal and S. Sahlin, "Use of desmopressin to prevent bleeding complications in patients treated with aspirin," British Journal of Surgery, vol. 80, no. 6, pp. 723-724, 1993.

[14] S. Schulman, "Pharmacologic tools to reduce bleeding in surgery," Hematology: The Education Program of the American Society of Hematology, vol. 2012, pp. 517-521, 2012.

[15] S. Lethagen, "Desmopressin (DDAVP) and hemostasis," Annals of Hematology, vol. 69, no. 4, pp. 173-180, 1994.

[16] F. Arzneimittel, Fachinformation MINIRIN Parenteral, 2011.

[17] I. F. K. C. Universitätsklinikum-Köln, Aufbau und Funktionsweise des PFA-100, 2012, http://www.unsere-uniklinik.de/institute/kchemie/Parameter.html.

[18] R. Karger, K. Reuter, J. Rohlfs, C. Nimsky, U. Sure, and V. Kretschmer, "The platelet function analyzer (PFA-100) as a screening tool in neurosurgery," ISRN Hematology, vol. 2012, Article ID 839242, 7 pages, 2012.

[19] H. Alahmadi, S. Vachhrajani, and M. D. Cusimano, "The natural history of brain contusion: an analysis of radiological and clinical progression: clinical article," Journal of Neurosurgery, vol. 112, no. 5, pp. 1139-1145, 2010.

[20] D. Kurland, C. Hong, B. Aarabi, V. Gerzanich, and J. M. Simard, "Hemorrhagic progression of a contusion after traumatic brain injury: a review," Journal of Neurotrauma, vol. 29, no. 1, pp. 1931, 2012.

[21] J. G. M. Van Beek, N. A. Mushkudiani, E. W. Steyerberg et al., "Prognostic value of admission laboratory parameters in traumatic brain injury: results from the IMPACT study," Journal of Neurotrauma, vol. 24, no. 2, pp. 315-328, 2007.

[22] S. Schulz-Stübner, D. Zielske, and R. Rossaint, "Comparison between nasal and intravenous desmopressin for the treatment of aminosalicylic acid-induced platelet dysfunction," European Journal of Anaesthesiology, vol. 19, no. 9, pp. 647-651, 2002.

[23] P. A. Kouides, V. R. Byams, C. S. Philipp et al., "Multisite management study of menorrhagia with abnormal laboratory haemostasis: a prospective crossover study of intranasal desmopressin and oral tranexamic acid," British Journal of Haematology, vol. 145, no. 2, pp. 212-220, 2009.

[24] M. C. Guth, L. Kaufner, C. Kleber, and C. von Heymann, "Therapy of trauma-induced coagulopathy-what is the evidence?" Anästhesiologie, Intensivmedizin, Notfallmedizin, Schmerztherapie, vol. 49, no. 9, pp. 528-540, 2012.

[25] R. A. Reiter, F. Mayr, H. Blazicek et al., "Desmopressin antagonizes the in vitro platelet dysfunction induced by GPIIb/IIIa inhibitors and aspirin," Blood, vol. 102, no. 13, pp. 4594-4599, 2003.

[26] J. S. Batchelor and A. Grayson, "A meta-analysis to determine the effect on survival of platelet transfusions in patients with either spontaneous or traumatic antiplatelet medicationassociated intracranial haemorrhage," BMJ Open, vol. 2, no. 2, Article ID e000588, 2012.

[27] G. Taylor, D. Osinski, A. Thevenin, and J.-M. Devys, "Is platelet transfusion efficient to restore platelet reactivity in patients who are responders to aspirin and/or clopidogrel before emergency surgery?" Journal of Trauma and Acute Care Surgery, vol. 74, no. 5, pp. 1367-1369, 2013.
[28] C. W. Washington, D. J. E. Schuerer, and R. L. Grubb, "Platelet transfusion: an unnecessary risk for mild traumatic brain injury patients on antiplatelet therapy," Journal of Trauma-Injury, Infection and Critical Care, vol. 71, no. 2, pp. 358-363, 2011.

[29] P. M. Mannucci and M. Levi, "Drug therapy: prevention and treatment of major blood loss," The New England Journal of Medicine, vol. 356, no. 22, pp. 2301-2311, 2007. 


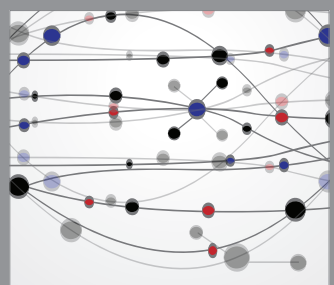

The Scientific World Journal
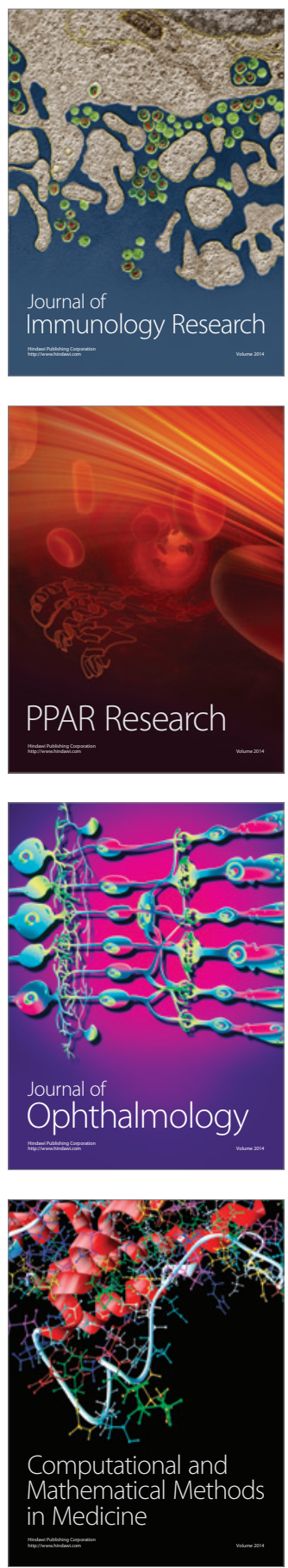

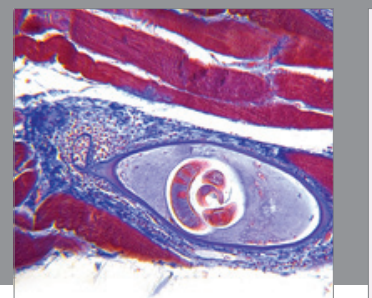

Gastroenterology

Research and Practice
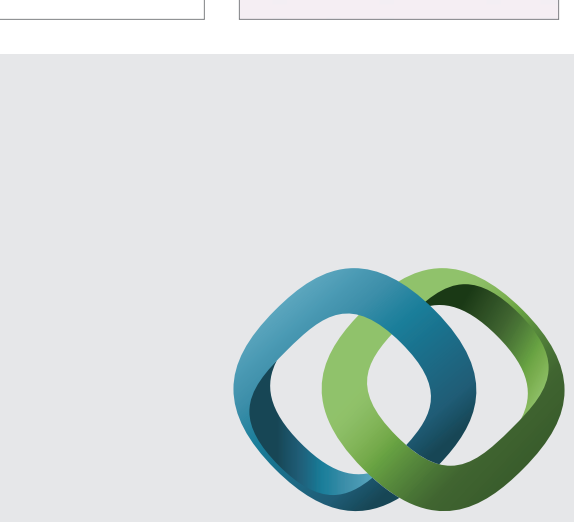

\section{Hindawi}

Submit your manuscripts at

http://www.hindawi.com
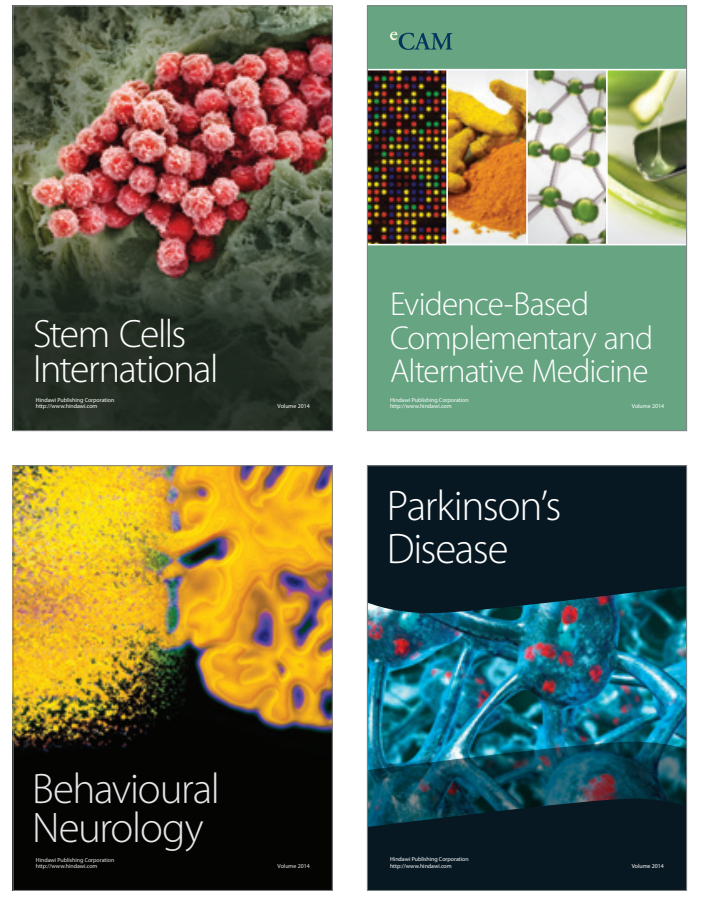
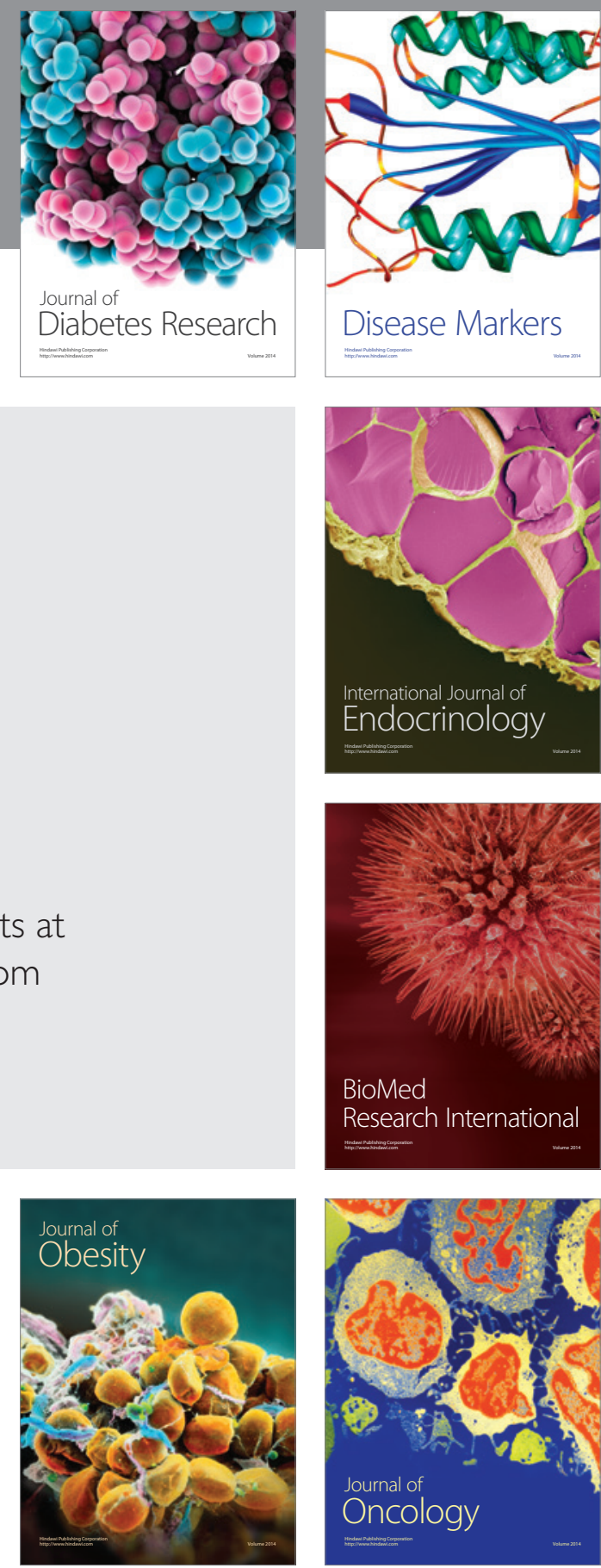

Disease Markers
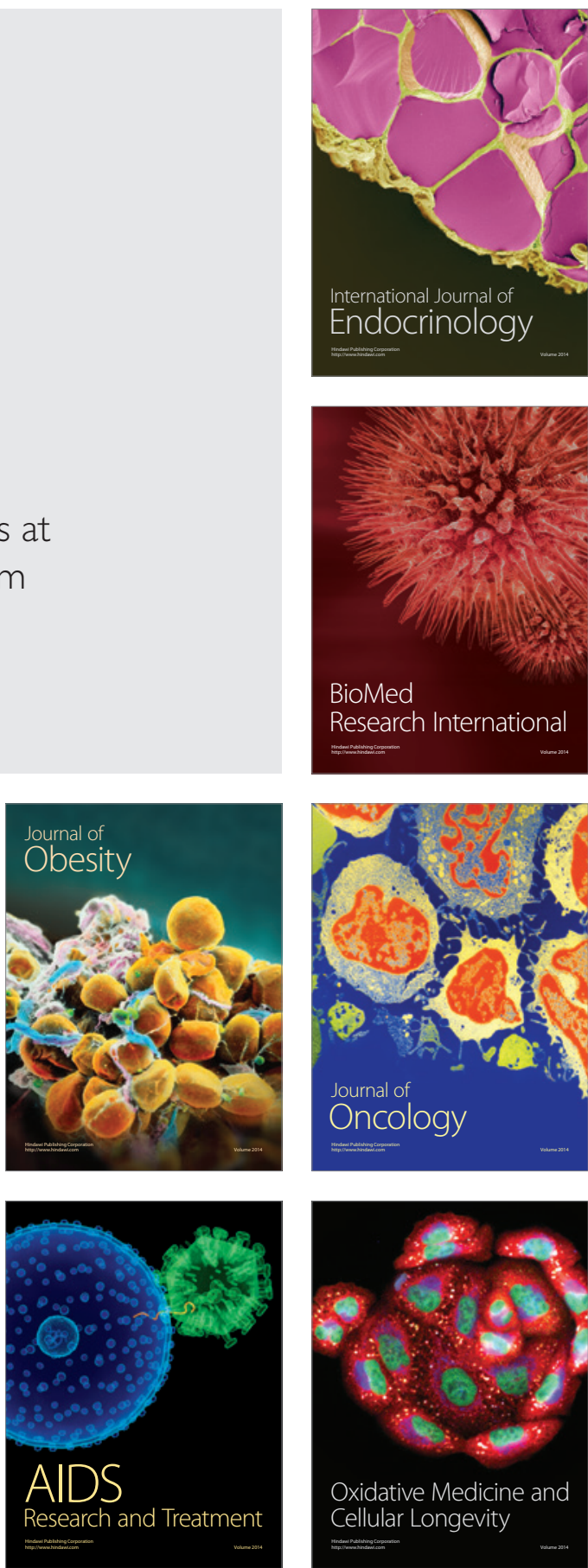